\title{
Left prefrontal cortex control of novel occurrences during recollection: A psychopharmacological study using scopolamine and event-related fMRI
}

\author{
M. Bozzali, ${ }^{\text {a,b,* }}$ S.E. MacPherson, ${ }^{\text {a }}$ R.J. Dolan, ${ }^{b}$ and T. Shallice ${ }^{a}$ \\ anstitute of Cognitive Neuroscience, University College London, UK \\ ${ }^{\mathrm{b}}$ Wellcome Department of Imaging Neuroscience, Institute of Neurology, University College of London, 12 Queen Square, London, WC1N $3 B G$, UK
}

Received 1 March 2006; revised 10 June 2006; accepted 15 June 2006

Available online 17 August 2006

Recollection and familiarity represent two processes involved in episodic memory retrieval. We investigated how scopolamine (an antagonist of acetylcholine muscarinic receptors) influenced brain activity during memory retrieval, using a paradigm that separated recollection and familiarity. Eighteen healthy volunteers were recruited in a randomized, placebo-controlled, double-blind design using eventrelated fMRI. Participants were required to perform a verbal recognition memory task within the scanner, either under placebo or scopolamine conditions. Depending on the subcondition, participants were required to make a simple recognition decision (old/new items) or base their decision on more specific information related to prior experience (target/non-target/new items). We show a drug modulation in left prefrontal and perirhinal cortex during recollection. Such an effect was specifically driven by novelty and showed an inverse correlation with accuracy performance. Additionally, we show a direct correlation between drug-related signal change in left prefrontal and perirhinal cortices. We discuss the findings in terms of acetylcholine mediation of the familiarity/novelty signal through perirhinal cortex and the control of the relative signal strength through prefrontal cortex.

(C) 2006 Elsevier Inc. All rights reserved.

Keywords: fMRI; Scopolamine; Episodic memory; Recollection; Familiarity

\section{Introduction}

The conscious experience of prior occurrence that accompanies human episodic memory retrieval in humans is complex, involving a number of different neuronal structures. It is widely believed that at least two types of processes are involved in episodic retrieval, namely recollection, the conscious experience of a previous event,

\footnotetext{
* Corresponding author. Wellcome Department of Imaging Neuroscience, Institute of Neurology, University College of London, 12 Queen Square, London, WC1N 3BG, UK. Fax: +44 02078131420.

E-mail address: m.bozzali@fil.ion.ucl.ac.uk (M. Bozalli).

Available online on ScienceDirect (www.sciencedirect.com).
}

and familiarity, where no contextual information is associated with the memory (Mandler, 1980; Hintzman et al., 1998; Yonelinas and Levy, 2002). Moreover, it is widely asserted that these processes have separable anatomical and neurochemical substrates within the medial temporal lobe involving hippocampus and perirhinal cortex respectively (Aggleton and Brown, 1999, 2005; Henson, 2005; but see Cipolotti et al., 2006). A recent study, using event-related brain potentials and midazolam (an amnesia inducing benzodiazepine), has provided evidence for separate processes underlying familiarity and recollection (Curran et al., 2006).

Recollection occurs inappropriately in the syndrome of confabulation, which typically arises from inferior medial prefrontal lesions, as for instance, following ruptured aneurysms of the anterior communicating artery. Some authors have argued for a double deficit account of confabulation, implicating episodic memory and executive functions (e.g. DeLuca and Cicerone, 1991; DeLuca, 1993; Fischer et al., 1995). However, meta-analyses of reports of positive cases (Gilboa and Moscovitch, 2002) do not support this hypothesis.

A second possible explanation can be derived by considering a structure frequently found to be damaged in patients who confabulate, namely the basal forebrain (Alexander and Freedman, 1984, Damasio et al., 1985). In the meta-analysis of Gilboa and Moscovitch (2002), they reported that $32 \%$ of the 79 confabulating patients had damage to this region and in 10 it was the sole lesion detected. The basal forebrain is the seat of four overlapping pathways of cholinergic projection systems. This led Damasio et al. (1985), when discussing a patient who manifested bizarre confabulation, to argue that "damage to the basal forebrain could lead to significant reduction of cholinergic input to temporal lobe structures and widespread regions of the association cortices. In both sites, the reduction might contribute to a memory defect" (p. 270). Two of the four basal forebrain cholinergic cell groups - Ch1 and Ch2 - project to the hippocampus (Mesulam, 2000). A theoretical approach to cholinergic modulation of the hippocampus developed by Hasselmo et al. (1996) suggests that these inputs act as a switch to move from processing retrieval to encoding mode. Thus, as far as the 
hippocampus is concerned, loss of cholinergic input would predict reduced capacity for encoding, whereas confabulation is generally viewed as a disorder of retrieval (Moscovitch, 1989; Burgess and Shallice, 1996). Two other principal cell groups in the basal forebrain include the $\mathrm{Ch} 4$ cell group, the major source of cholinergic input to the cortex. One possibility is that a lesion to this system gives rise to confabulation. Such a hypothesis could explain why a patient with a severe amnesia, as reported by Phillips et al. (1987), with selective damage to the basal forebrain might not confabulate; the nucleus basalis, the site of origin of $\mathrm{Ch} 4$ neurons, was spared in this patient.

What then are the most critical cortical regions, as far as the cholinergic input is concerned, with respect to confabulation? The re-experience of prior episodes and its impairment in confabulation has been suggested to involve a complex interplay between online core memory retrieval systems involving medial temporal regions and prefrontal control systems (e.g. Moscovitch, 1989). This potential involvement of prefrontal cortex is consistent with imaging studies of episodic memory retrieval that demonstrate extensive prefrontal activation (Tulving et al., 1994; Shallice et al., 1994; Lepage et al., 2000; Fletcher and Henson, 2001). Moreover, it is known from a number of studies that loss of cholinergic input leads to reduced activation in prefrontal structures (e.g. Chudasama et al., 2004; Sperling et al., 2002).

One hypothesis for the role of dorsolateral prefrontal cortex in memory retrieval, particularly in the right hemisphere, is that it concerns monitoring and checking the products of the ecphoric process (Fletcher and Henson, 2001; Shallice, 2002; Cabeza et al., 2003). One possibility is that these structures are deactivated as a result of loss of cholinergic input to the cortex, and this leads to impairment in the monitoring of retrieval errors. This can occur especially if there are additional memory problems with consequent production of confabulations. To test such a hypothesis, we investigated the effect of scopolamine on a complex memory retrieval task that strongly activates dorsolateral prefrontal cortex.

Scopolamine is a powerful antagonist of the muscarinic Ach receptors and blocks central cholinergic transmission. To our knowledge, Mintzer and Griffiths (2001) have conducted the only study to investigate the effect of scopolamine on retrieval using a paradigm separating recollection and familiarity processes. They examined the effects of low and high doses of scopolamine on the so-called Deese-Roediger false recognition paradigm (see Roediger and McDermott, 1995). For low doses of scopolamine, recollection responses were reduced for correct recognition but were unaffected for the confabulatory so-called 'theme' recollection responses. In contrast, familiarity responses were unaffected. Thus, a higher proportion of recollection responses were spurious. At higher scopolamine dosages, all types of retrieval responses were reduced.

The task we employed in this study was adapted from that used by Henson et al. (1999a), in turn based on the Jacoby exclusion task (Jacoby, 1996). This task was chosen for two reasons. First, the contrast between Exclusion and Inclusion conditions potentially allows testing of differential effects of scopolamine on recollection (source memory) and familiarity to be examined. Second, as shown by Henson et al. (1999a), the paradigm leads to extensive activation of lateral prefrontal cortex, both dorsolateral and ventrolateral, particularly on the right, which are likely to test $\mathrm{Ch} 4$ cholinergic target projection regions.

We recruited a group of young healthy volunteers to study the effect of scopolamine on this task. Memory retrieval abilities were separately tested under two different conditions: with and without the administration of scopolamine. The aim was to explore which brain regions are modulated by the cholinergic inputs during memory recollection. As we were concerned with the effects of scopolamine on a complex retrieval task and as scopolamine influences the efficiency of encoding, we administered the drug after the study phase, which meant that there was a much longer study-test gap compared with the Henson et al. (1999a) study. Furthermore, for simplicity, source memory requirements were solely based on spatial position, color and font size.

\section{Methods}

\section{Subjects}

The present event-related functional MR imaging study involved a group of 18 right-handed, native English-speaking healthy volunteers ( 11 women and 7 men; mean age $=25.9$ years; $\mathrm{SD}=4.3$; range $=19-33$ ) with no history of medical or psychiatric disorders. All were recruited from a pool of psychology students. Each volunteer participated in two different sessions (drug and placebo randomly appointed) separated by a 7- to 10-day interval. Ethical approval from the Joint Ethics Committee of the National Hospital for Neurology and Neurosurgery and the Institute of Neurology and written informed consent were obtained before study initiation.

\section{Experimental procedure and cognitive paradigm}

For each session (drug or placebo), the cognitive task involved the following two experimental conditions: (1) Encoding (study) and (2) Recognition (test) phase. The recognition phase was split in two different subconditions: (2a) inclusion (I), requiring a simple recognition decision; (2b) exclusion (E), involving a judgment based on the retrieval of more specific information related to prior experience (context).

After the study phase, $0.4 \mathrm{mg}$ of scopolamine or saline (placebo) was randomly administered to each subject using a double-blinded placebo-controlled drug administration technique. Each subject received an intravenous cannula into his/her left cubital fossa, and an infusion of either scopolamine or saline was administered, depending on the session. All subjects had their blood pressure and pulse frequency checked after injection and were given questionnaires to document subjective ratings of potential side effects. These questionnaires included the subjective feelings reported in the Bond and Larder scale (Bond and Lader, 1974) and three additional adverse reactions associated with scopolamine (dizziness, dry mouth and blurred vision). Subjects were asked to score each item ranging from 0 (absence of any abnormality) to 6 (abnormality strongly present). Each participant was scanned 90 min after injection, with an event-related fMRI paradigm of the recognition task.

In each condition, the presented words were 4- to 9-letter nouns with imageability values ranging from 550 to 700 (MRC Psycholinguistic Database; http://www.psy.uwa.edu.au/mrcdatabase/ uwa $\_$rc.htm). The timing of all the events for each trial in the test phase is schematically summarized in Fig. 1. The encoding condition was conducted outside the scanner using a laptop. It was characterized by the visual presentation of one hundred and twenty sequential low and medium frequency [T-LFRQ $<150$ ] words (four matched blocks with 30 -item repetition each). Each item was 


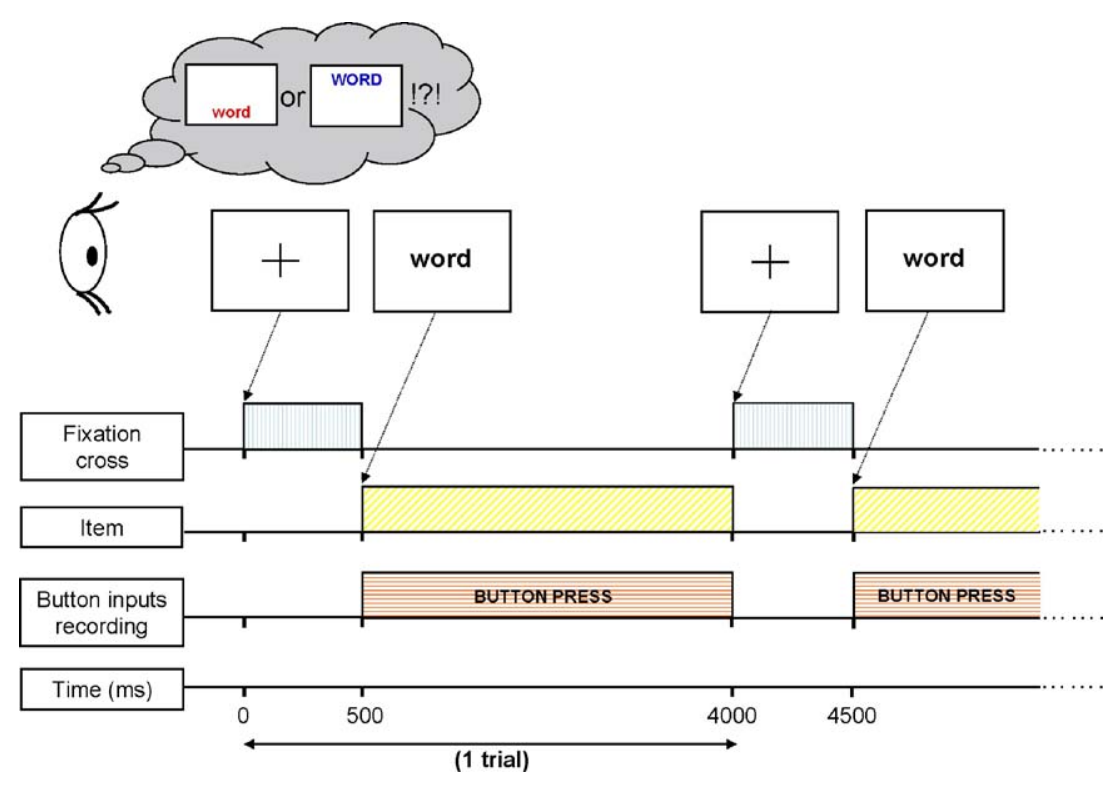

Fig. 1. The fMRI paradigm used during retrieval is schematically represented. In each trial, a black colored word positioned in the center of the computer screen was presented for $3.5 \mathrm{~s}$. Between each word presentation, subjects were asked to focus on a central fixation cross. Null events (a black cross positioned in the center of a white screen for the duration of one trial) were randomly intermixed with actual trials. During the inclusion condition, subjects were requested to judge, by button pressing, whether the presented word had already been seen (target items) or not (new items) during the encoding phase. During the exclusion condition, subjects were asked to press a button both if the word was old and had previously been presented in one of the two contexts (target items) and another button in all other cases (non-target and new items). See Methods for further details.

randomly presented for $3.5 \mathrm{~s}$ in association with one of two equally frequent possible contextual patterns (large sized word in blue appearing at the top or small sized word in red appearing at the bottom of the screen). The subjects had to memorize both the word and its contextual pattern (position/color/size). The block presentation was randomly ordered.

The recall phase was conducted inside the scanner during fMRI data acquisition. The subjects were presented with four pseudorandomly selected blocks (two for each subcondition, inclusion and exclusion) with 45 centrally presented words colored black positioned in the center of the screen. One hundred and twenty words (60 low and 60 medium frequency) had been already presented in the study phase (half in each encoding pattern), and sixty were new words. In each block, 15 null events (a black cross positioned in the center of a white screen for the duration of one trial) were randomly interspersed. One of the following two schemes was randomly adopted in each experiment: (1) IEEI; (2) EIIE.

During the inclusion condition of the recall phase, the subjects were requested to judge, by pressing one of two buttons, whether the presented word had already been seen in the encoding phase (target items) or not (new items). During the exclusion phase, subjects were asked to decide whether the word had already been presented during the encoding phase with a given contextual pattern (target) or not (non-target and new items).

\section{Imaging and image processing}

MRI data were acquired from a 3 T Allegra system (Siemens, Erlangen, Germany). Functional images were collected by echoplanar T2* sequence using BOLD (blood-oxygenation-level-dependent) contrast. Each acquired volume consisted of 40 axial slices with a $2.0 \mathrm{~mm}$ thickness and a $0.5 \mathrm{~mm}$ distance factor in order to cover the entire brain, with an effective repetition time of $2.6 \mathrm{~s}$. The scanner was synchronized with the presentation of each block, and the ratio of inter-scan to inter-stimulus interval ensured that voxels were sampled at different phases relative to stimulus onset. The first five volumes were discarded to allow for T1 equilibration effects. In order to correct for geometric distortions that particularly affect EPI images acquired at high magnetic field, we also collected at the beginning of each study a fieldmap based on a set of dual echo-time images $\left(\mathrm{TE}_{1}=29 \mathrm{~ms}\right.$, $\mathrm{TE}_{2}=19 \mathrm{~ms}$ ). Each EPI fieldmap was processed (Hutton et al., 2002) using the SPM2 fieldmap toolbox to produce a voxel displacement map indicating the static field distortions. All the acquired EPI images were then realigned to the first image of the first session using the 'Realign + Unwrap' routine in SPM2, including the calculated voxel displacement map. This procedure corrected for distortions at each time point by combining the measured fieldmap with the estimated changes in field due to head motion, as described by Hutton et al. (2004). The EPI images were then normalized to a standard echoplanar image template and smoothed with a Gaussian kernel of $8 \mathrm{~mm}$ full-width half maximum.

\section{Statistical analysis of images}

Data were processed using MATLAB 6.5 (MathWork, Natick, MA) and SPM2 (Wellcome Dept. Cogn. Neurol., London; http:// www.fil.ion.ucl.ac.uk/spm) and analyzed with a general linear model for event-related designs, using a random-effects analysis. Data were then globally scaled and high-passed-filtered to 1/ $128 \mathrm{~Hz}$ to remove low-frequency noise. For each subject and session (drug or placebo), the following events were modeled separately using the button press time as onset and dividing each of them in events that obtained correct or incorrect answers: (a) inclusion target items; (b) inclusion new items; (c) exclusion target items; (d) exclusion non-target items; (e) exclusion new items. In 
those trials in which the answer was missed (separately grouped), the average reaction time calculated over the entire block was used as onset time. The same conditions modeled in the single subject analysis were included in the random-effects analysis using a oneway analysis of variance (ANOVA) design. The baseline included the null events and the cross fixation, which separated each trial from the following one. Neither was modeled.

To limit the number of statistical tests and the attendant risk of false-positives, the overall BOLD effects related with the inclusion and exclusion task for each session (placebo or scopolamine) were investigated by collapsing the different subconditions modeled: (1) inclusion condition vs. baseline; (2) exclusion condition vs. baseline; (3) inclusion condition vs. exclusion condition; (4) exclusion condition vs. inclusion condition. The effect of the scopolamine, which represents the main interest in the present study, was separately investigated for inclusion (inclusion-placebo vs. exclusion-placebo $>$ inclusion-scopolamine vs. exclusion-scopolamine) and exclusion (exclusion-placebo vs. inclusion-placebo > exclusion-scopolamine vs. inclusion-scopolamine). For the comparisons investigating activation versus baseline, we report $P_{\text {corrected }}<0.05$, while differential effects and interactions are reported at $P_{\text {uncorrected }}<0.001$. Stereotaxic coordinates are reported in Talairach space and correspond to the standard MNI brain (Cocosco et al., 1997). These coordinates bear a close, but not exact, match to the atlas of Talairach and Tournoux (1998).

To investigate the possible correlations between individual subject behavioral performance and the activation changes of those areas that have been found to be modulated by scopolamine, the Spearman Rank Correlation Coefficient was used. To correct for multiple comparisons and to minimize the risk of type II errors, a Bonferroni correction was applied and only $P$ values $\leq 0.0008$ were considered statistically significant. For each subcondition (target and new for inclusion, and target, non-target and new for exclusion), the percentage of correct answers was correlated with the change in activation (expressed as the percentage difference from the mean value calculated over the entire population of studied subjects).

\section{Results}

Accuracy and reaction time data

None of the subjects reported side effects after placebo administration. However, 3 of the 18 subjects were excluded from the analysis due to excessive sedation during the drug session. After scopolamine administration, they expressed a decrease in alertness $($ mean $=4.67 ; \mathrm{SD}=0.58)$, a moderate dry mouth $($ mean $=3.67$;
$\mathrm{SD}=0.44)$ and dizziness (mean $=3.41 ; \mathrm{SD}=0.54)$ on a scale of 0 to 6 . All other subjects, under scopolamine, reported modest side effects (ranging from 1 to 2 ) that included a dry mouth and dizziness only. None of them reported a change in alertness. The performance of the remaining 15 subjects was analyzed separately for the inclusion and exclusion conditions. A 2 (drug vs. placebo) $\times 2$ (target vs. new) repeated-measures ANOVA was conducted on the inclusion data, and a separate 2 (drug vs. placebo) $\times 3$ (target vs. non-target vs. new) ANOVA was conducted on the exclusion data. Post hoc analyses were performed using Bonferroni $t$ tests.

In the inclusion condition, a $2 \times 2$ repeated-measures ANOVA demonstrated a significant main effect of response $[F(1,14)=23.40$, $P<0.0001]$ where new responses were identified significantly more accurately than target responses. There was no effect of drug or response $\times$ drug interaction $[F(1,14)<0.34]$. In the exclusion condition, a $2 \times 3$ ANOVA revealed a significant main effect of response $[F(2,28)=11.56, P<0.0001]$ and post hoc analysis showed that new items were correctly identified significantly better than non-targets and targets. There was no main effect of drug $[F(1,14)=0.17$ or response $\times$ drug interaction $[F(2,28)=0.17]$.

In terms of the reaction time data, a 2 (drug vs. placebo) $\times 2$ (inclusion vs. exclusion) $\times 2$ (old vs. new) repeated-measures ANOVA was conducted. There was a significant main effect of condition $[F(1,14)=41.36, P<0.0001]$ where correct responses in the exclusion condition were made significantly more slowly than correct responses in the inclusion condition. There was also a significant condition $\times$ response interaction $[F(1,14)=71.34, P<0.0001]$. Post hoc analysis using Bonferroni $t$ tests demonstrated that the response times for correctly identified old items (targets and non targets) were significantly slower in the exclusion condition than the inclusion condition. However, the response times for correctly identified new items did not significantly differ across conditions. There was no main effect of drug $[F(1,14)=0.26]$ or response $[F(1,14)=0.33]$ or drug $\times$ response $[F(1,14)=0.30]$, drug $\times$ condition $[F(1,14)=0.29]$ or drug $\times$ condition $\times$ response interaction $[F(1,14)=0.70]$. Accuracy and reaction times (RT) data are shown in Table 1.

\section{Imaging findings}

\section{Placebo session}

Contrasting the inclusion condition against the baseline, we observed activation in a number of different regions (predominantly left lateralized) that survived after correction for multiple comparisons $\left(P_{\mathrm{FWE}}\right.$ corrected $\left.<0.05\right)$. This activation response is summarized in Table 2.

Contrasting the exclusion condition against the baseline, a much more widespread pattern of activation (again, predominantly

Table 1

Mean percentage accuracy and mean correct reaction time (s) by session (placebo or scopolamine), condition (inclusion or exclusion) and item type (target, new, non-target)

\begin{tabular}{|c|c|c|c|c|c|c|c|c|c|c|c|}
\hline & & \multicolumn{5}{|l|}{ Placebo } & \multicolumn{5}{|c|}{ Scopolamine } \\
\hline & & \multicolumn{2}{|c|}{ Inclusion } & \multicolumn{3}{|c|}{ Exclusion } & \multicolumn{2}{|c|}{ Inclusion } & \multicolumn{3}{|c|}{ Exclusion } \\
\hline & & Targets & New & Targets & New & Non-targets & Targets & New & Targets & New & Non-targets \\
\hline \multirow[t]{2}{*}{ Correct } & Mean & 80.62 & 91.32 & 82.06 & 92.91 & 86.54 & 82.28 & 92.26 & 81.98 & 93.84 & 89.00 \\
\hline & SD & 13.26 & 10.24 & 12.64 & 12.03 & 13.07 & 10.59 & 6.34 & 15.71 & 5.45 & 7.93 \\
\hline \multirow[t]{2}{*}{ Reaction time } & Mean & 1.22 & 1.32 & 1.33 & 1.35 & 1.56 & 1.24 & 1.33 & 1.51 & 1.35 & 1.54 \\
\hline & SD & 0.19 & 0.22 & 0.20 & 0.25 & 0.25 & 0.25 & 0.27 & 0.35 & 0.24 & 0.19 \\
\hline
\end{tabular}

See text for further details. 
Table 2

Maxima of regions showing significant $(P<0.05$ corrected) BOLD signal changes in comparison of inclusion and baseline condition

\begin{tabular}{|c|c|c|c|c|c|c|c|}
\hline \multirow[t]{2}{*}{ Brain region } & \multirow[t]{2}{*}{ Side } & \multirow[t]{2}{*}{$\mathrm{BA}$} & \multirow[t]{2}{*}{ Size } & \multicolumn{3}{|c|}{$\begin{array}{l}\text { Coordinates } \\
(\mathrm{mm})\end{array}$} & \multirow{2}{*}{$\begin{array}{l}\text { Peak } \\
Z \\
\text { score }\end{array}$} \\
\hline & & & & $x$ & $y$ & $z$ & \\
\hline Mid occipital gyrus & $\mathrm{L}$ & $17,18,19$ & 2977 & -30 & -88 & -20 & 7.59 \\
\hline Fusiform gyrus & $\mathrm{L}$ & 37 & & -40 & -53 & -22 & 6.57 \\
\hline Cerebellum & $\mathrm{L}$ & - & & -39 & -54 & -32 & 7.29 \\
\hline Mid/inf occipital gyrus & $\mathrm{R}$ & $17,18,19$ & 2582 & 36 & -85 & -13 & 6.07 \\
\hline Fusiform gyrus & $\mathrm{R}$ & 37 & & 36 & -54 & -25 & 5.69 \\
\hline Cerebellum & $\mathrm{R}$ & - & & 36 & -82 & -24 & 7.55 \\
\hline Cerebellum & $\mathrm{R}$ & - & 25 & 8 & -80 & -38 & 5.07 \\
\hline Inf parietal lobule & $\mathrm{L}$ & 5,7 & 723 & -48 & 38 & 48 & 5.36 \\
\hline Supramarginal gyrus & $\mathrm{L}$ & 40 & & -49 & -38 & 42 & 4.69 \\
\hline Postcentral gyrus & $\mathrm{L}$ & $2,3,4$ & & -36 & -30 & 50 & 6.98 \\
\hline Precentral gyrus & $\mathrm{L}$ & 4 & & -42 & -24 & 58 & 5.35 \\
\hline $\begin{array}{l}\text { Sup and inf parietal } \\
\text { lobule }\end{array}$ & $\mathrm{L}$ & 7 & 31 & -36 & -58 & 56 & 5.32 \\
\hline Sup temporal gyrus & $\mathrm{L}$ & 38 & & -48 & 15 & -10 & 5.01 \\
\hline Inf frontal gyrus & $\mathrm{L}$ & 45,47 & 544 & -34 & 22 & -4 & 6.99 \\
\hline Insula & $\mathrm{L}$ & - & & -42 & 12 & -2 & 5.44 \\
\hline Inf frontal gyrus & $\mathrm{R}$ & 47 & 370 & 34 & 26 & -8 & 6.67 \\
\hline Insula & $\mathrm{R}$ & - & & 35 & 19 & 6 & 5.45 \\
\hline Med frontal gyrus & $\mathrm{L}$ & 6,8 & & -8 & 5 & 50 & 4.91 \\
\hline Ant cingulate & $\mathrm{L}$ & 24,32 & & -5 & 14 & 40 & 5.81 \\
\hline Med frontal gyrus & $\mathrm{R}$ & 6,8 & 966 & 2 & 16 & 44 & 6.97 \\
\hline Sup frontal gyrus & $\mathrm{R}$ & 6,8 & & 8 & 6 & 61 & 4.95 \\
\hline Ant cingulate & $\mathrm{R}$ & 32 & & 9 & 19 & 34 & 4.89 \\
\hline Inf frontal gyrus & $\mathrm{L}$ & $9,44,45$ & 376 & -48 & 6 & 28 & 6.45 \\
\hline Thalamus & $\mathrm{L}$ & - & 75 & -12 & -20 & 4 & 5.12 \\
\hline Mammillary body & $\mathrm{L}$ & - & 59 & -12 & -18 & 1 & 4.94 \\
\hline Subthalamic nucleus & $\mathrm{L}$ & - & & -11 & -1 & -5 & 4.96 \\
\hline
\end{tabular}

Abbreviations: $\sup =$ superior; inf $=$ inferior; $a n t=$ anterior; post $=$ posterior; mid $=$ middle; med $=$ medial $; \mathrm{R}=$ right $\mathrm{L}=$ left $\mathrm{B}=$ bilateral $; \mathrm{BA}=$ Brodmann area.

The extension of each area (size) is expressed in number of voxels. Only regions that were statistically significant at uncorrected level $\left(P_{\text {corrected }}<0.05\right)$ with a size of 10 or more voxels have been considered. See text for further details.

left lateralized) than that obtained in the inclusion condition was found (see Table 3).

Contrasting the inclusion with exclusion condition (exclusion minus inclusion) revealed significant effects. These included bilateral medial frontal gyrus (BA6), anterior (BA 24, 32) and posterior cingulate (BA 23,31), precuneus (BA 7), the angular gyrus (BA 40), occipital lobe (BA 18,19) and cerebellum. There also was the unilateral involvement of the left supramarginal gyrus (BA 39), pre- (BA 4, 6) and postcentral (BA 1, 2, 3) gyri, frontal gyri (BA 8, 9, 11), the left fusiform gyrus (BA 20, 37) and the inferior parietal lobe (BA 7). Additional subcortical activations were noted in the region of the left red nucleus and substantia nigra (see Table 4).

\section{Drug effects}

The interaction between drug and inclusion/exclusion condition (inclusion-placebo vs. exclusion-placebo > inclusion-scopolamine vs. exclusion-scopolamine) was significant in a number of regions. The interaction effects between drug and inclusion condition (inclusion-placebo vs. exclusion-placebo > inclusion-scopolamine vs. exclusion-scopolamine) indicated that a few regions were modulated by scopolamine in the inclusion task compared to the exclusion task, most notably the left inferior frontal gyrus (BA 9, 45) and left cerebellum.

Conversely, the opposite interaction showed scopolamine modulated activity in several regions. These regions include the medial frontal gyri (BA6) bilaterally, the left superior (BA9) and middle frontal gyrus (BA 6), the right medial frontal gyrus (BA 8), the left anterior (BA 24) and right posterior (BA 23,31) cingulate, the left perirhinal cortex (BA 36), the left superior

Table 3

Maxima of regions showing significant $(P<0.05$ corrected $)$ BOLD signal changes in comparison of exclusion and baseline condition

\begin{tabular}{|c|c|c|c|c|c|c|c|}
\hline \multirow[t]{2}{*}{ Brain region } & \multirow[t]{2}{*}{ Side } & \multirow[t]{2}{*}{ BA } & \multirow[t]{2}{*}{ Size } & \multicolumn{3}{|c|}{$\begin{array}{l}\text { Coordinates } \\
(\mathrm{mm})\end{array}$} & \multirow{2}{*}{$\begin{array}{l}\text { Peak } \\
Z \\
\text { score }\end{array}$} \\
\hline & & & & $x$ & $y$ & $z$ & \\
\hline Inf/mid occipital gyrus & $\mathrm{L}$ & $17,18,19$ & 12,258 & -26 & -96 & -6 & 8.16 \\
\hline Cuneus & $\mathrm{L}$ & $17,18,19$ & & -9 & -96 & -11 & 7.06 \\
\hline Fusiform gyrus & $\mathrm{L}$ & 37 & & -38 & -64 & -16 & 7.01 \\
\hline Cerebellum & $\mathrm{L}$ & - & & -31 & -63 & -38 & 5.69 \\
\hline Inf/mid occipital gyrus & $\mathrm{R}$ & 18,19 & & 32 & -87 & -10 & 7.46 \\
\hline Fusiform gyrus & $\mathrm{R}$ & 37 & & 40 & -56 & -20 & 4.83 \\
\hline Cuneus & $\mathrm{R}$ & 18,19 & & 11 & -73 & 3 & 6.19 \\
\hline Cerebellum & $\mathrm{R}$ & - & & 23 & -52 & -32 & 6.84 \\
\hline Inf parietal lobule & $\mathrm{L}$ & 7 & 11,148 & -34 & -30 & 50 & 7.98 \\
\hline Precuneus & $\mathrm{L}$ & 7 & & -6 & -76 & 30 & 5.19 \\
\hline Precuneus & $\mathrm{R}$ & 7 & & 10 & -75 & 36 & 5.92 \\
\hline Sup parietal lobule & $\mathrm{L}$ & 5,7 & & -34 & -65 & 46 & 7.04 \\
\hline Angular gyrus & $\mathrm{L}$ & 39 & & -36 & -70 & 34 & 5.76 \\
\hline Supramarginal gyrus & $\mathrm{L}$ & 40 & & -37 & -61 & 53 & 7.53 \\
\hline Pre/postcentral gyrus & $\mathrm{L}$ & $1,2,3,4$ & & -35 & -53 & 51 & 6.84 \\
\hline Med frontal gyrus & $\mathrm{L}$ & 8,9 & & -2 & 6 & 55 & 6.89 \\
\hline Mid frontal gyrus & $\mathrm{L}$ & 8,9 & & -14 & 1 & 54 & 4.81 \\
\hline Cingulate gyrus & $\mathrm{L}$ & 24,32 & & 10 & 12 & 34 & 5.46 \\
\hline Cingulate gyrus & $\mathrm{R}$ & 24,32 & & -5 & 6 & 37 & 6.50 \\
\hline Post cingulate & $\mathrm{L}$ & $23,29,31$ & 615 & -2 & -34 & 24 & 7.38 \\
\hline Post cingulate & $\mathrm{R}$ & $23,29,31$ & & 3 & -32 & 21 & 7.35 \\
\hline $\mathrm{Mid} /$ inf frontal gyrus & $\mathrm{L}$ & 10 & 80 & -44 & 48 & -4 & 5.26 \\
\hline Mid frontal gyrus & $\mathrm{L}$ & 9,10 & 37 & -38 & 42 & 30 & 5.24 \\
\hline Inferior frontal gyrus & $\mathrm{L}$ & $11,45,47$ & 2963 & -30 & 24 & -6 & 7.87 \\
\hline Insula & $\mathrm{L}$ & - & & -34 & 21 & 6 & 7.84 \\
\hline Parahippocampal gyrus & $\mathrm{L}$ & $27,28,35$ & 2723 & -23 & -30 & -10 & 7.82 \\
\hline Thalamus & $\mathrm{L}$ & - & & -13 & -18 & 2 & 5.48 \\
\hline Substantia nigra & $\mathrm{L}$ & - & & -9 & -12 & -13 & 5.26 \\
\hline Caudate head & $\mathrm{L}$ & - & & -14 & 8 & 10 & 5.20 \\
\hline Globus pallidus & $\mathrm{L}$ & - & & -16 & 6 & 1 & 4.89 \\
\hline Mammillary body & $\mathrm{L}$ & - & & -12 & -17 & 2 & 4.87 \\
\hline $\begin{array}{l}\text { Transversetemporal } \\
\text { gyrus }\end{array}$ & $\mathrm{L}$ & 41 & 81 & -48 & -26 & 16 & 5.22 \\
\hline Inf frontal gyrus & $\mathrm{R}$ & 47 & 617 & 36 & 24 & -8 & 7.82 \\
\hline Insula & $\mathrm{R}$ & - & & 34 & 19 & 6 & 7.81 \\
\hline Putamen & $\mathrm{R}$ & - & 334 & 12 & 14 & 2 & 5.38 \\
\hline Globus pallidus & $\mathrm{R}$ & - & & 15 & 7 & 1 & 4.88 \\
\hline Mammillary body & $\mathrm{R}$ & - & & 11 & -15 & 1 & 4.79 \\
\hline Caudate head & $\mathrm{R}$ & - & & 14 & 14 & 11 & 5.10 \\
\hline Thalamus & $\mathrm{R}$ & - & & 14 & -10 & 16 & 5.26 \\
\hline
\end{tabular}

Abbreviations: $\sup =$ superior; inf $=$ inferior; ant $=$ anterior; post $=$ posterior; mid $=$ middle; med $=$ medial; $\mathrm{R}=$ right; $\mathrm{L}=$ left; $\mathrm{B}=$ bilateral; $\mathrm{BA}=$ Brodmann area.

The extension of each area (size) is expressed in number of voxels. Only regions that were statistically significant at uncorrected level $\left(P_{\text {corrected }}<0.05\right)$ with a size of 10 or more voxels have been considered.

See text for further details. 
Table 4

Maxima of regions showing significant $(P<0.001$ uncorrected) BOLD signal increases in exclusion compared to inclusion condition under placebo

\begin{tabular}{lllll}
\hline Brain region & Side BA & Size $\begin{array}{l}\text { Coordinates } \\
(\mathrm{mm})\end{array}$ & $\begin{array}{l}\text { Peak } \\
\end{array}$ \\
& & & \\
& & & \\
& & &
\end{tabular}

\begin{tabular}{|c|c|c|c|c|c|c|c|}
\hline Precuneus & $\mathrm{L}$ & 7 & 949 & -6 & -64 & 38 & \\
\hline Posterior cingulate & $\mathrm{R}$ & 31 & & 14 & -62 & 26 & 4. \\
\hline Precuneus & $\mathrm{R}$ & 7 & & 10 & -68 & 42 & 4. \\
\hline Inf/sup parietal lobule & $\mathrm{L}$ & 7 & 1674 & -48 & -42 & 50 & 4.2 \\
\hline Angular gyrus & $\mathrm{L}$ & 40 & & & & & \\
\hline Supramarginal gyrus & $\mathrm{L}$ & 39 & & & & & \\
\hline Precentral gyrus & $\mathrm{L}$ & 4,6 & & -38 & -24 & 60 & \\
\hline ostcentral gyrus & $\mathrm{L}$ & $1,2,3$ & & -32 & -30 & 52 & \\
\hline erebellum & $\mathrm{R}$ & & 263 & 40 & -64 & 36 & \\
\hline Cingulate gyrus & $\mathrm{L}$ & 24,32 & 300 & -6 & 8 & 42 & \\
\hline Cingulate gyrus & $\mathrm{R}$ & 32 & & 6 & 9 & 43 & \\
\hline Med fronta & $\mathrm{L}$ & $6,8,9$ & & -4 & 26 & 38 & \\
\hline Media & $\mathrm{R}$ & 6 & & 4 & 14 & 47 & \\
\hline $\mathrm{Inf} / \mathrm{mic}$ & $\mathrm{L}$ & 18 & 176 & -26 & -96 & -6 & \\
\hline Red nucleus & $\mathrm{L}$ & - & 35 & -8 & -22 & -8 & 3. \\
\hline ubstantia nigra & $\mathrm{L}$ & & & -10 & -14 & -11 & 3. \\
\hline up $f$ & $\mathrm{~L}$ & 6 & 127 & -26 & -2 & 62 & 3. \\
\hline yrus & $\mathrm{L}$ & 6,9 & 108 & -48 & 8 & 28 & \\
\hline Inferior frontal gyr & $\mathrm{L}$ & 6,9 & & -50 & 4 & 38 & \\
\hline Inf/mid occipital gyrus & $\mathrm{R}$ & 17,18 & 61 & 26 & -92 & -4 & \\
\hline Ceret & $\mathrm{R}$ & - & 26 & 34 & -44 & 34 & \\
\hline Post ci & $\mathrm{R}$ & 23,31 & 53 & 2 & -40 & 24 & \\
\hline & $\mathrm{L}$ & 23,31 & & -1 & -36 & 24 & \\
\hline Cere & $\mathrm{L}$ & - & 38 & -38 & -48 & -30 & 3. \\
\hline Fusiform gyrus & $\mathrm{L}$ & 37 & & -38 & -44 & 21 & \\
\hline Inf-temporal/fusiform gyrus & $\mathrm{L}$ & 20,37 & 88 & -48 & -64 & -18 & \\
\hline Middle occipital gyrus & $\mathrm{L}$ & 19 & & -48 & -74 & -14 & \\
\hline Posterior & $\mathrm{L}$ & 11 & 11 & -28 & 26 & -8 & \\
\hline rbital gyrus & $\mathrm{L}$ & 11 & 5 & -40 & 38 & -20 & \\
\hline Cereb & $\mathrm{R}$ & - & 39 & 4 & -70 & -22 & \\
\hline & $\mathrm{L}$ & 6 & 15 & -30 & 6 & 50 & \\
\hline Angular gyrus & $\mathrm{R}$ & 40 & 10 & 44 & -44 & 54 & \\
\hline & $\mathrm{R}$ & 7 & 33 & 38 & -80 & 36 & \\
\hline Middle occipital gyrus & $\mathrm{R}$ & 18,19 & 37 & 34 & -84 & -18 & \\
\hline
\end{tabular}

Abbreviations: $\sup =$ superior; $\inf =$ inferior; ant $=$ anterior; post $=$ posterior; mid = middle; med =medial; $\mathrm{R}=$ right; $\mathrm{L}=$ left; $\mathrm{B}=$ bilateral; $\mathrm{BA}=$ Brodmann area.

The extension of each area (size) is expressed in number of voxels. Only regions that were statistically significant at uncorrected level $\left(P_{\text {uncorrected }}<0.001\right)$ with a size of 10 or more voxels have been considered.

See text for further details.

temporal gyrus (BA 22), the right inferior parietal lobule (BA5, 7) and cerebellum (see Table 5A). Investigation of the simple main effect (inclusion minus exclusion) under scopolamine revealed a similar pattern of drug modulation to that observed in the interaction between drug and exclusion condition. Such results are summarized in Table 5B. The same contrast under placebo did not reveal any significant difference. Examination of parameter estimates indicated that these effects were driven by responses to new items, as shown in Fig. 2. To further investigate such an effect and better address its interpretation, contrasts between old and new items were separately performed for each task (inclusion and exclusion), both under placebo and scopolamine. Consistent with the results obtained from the interaction between drug and exclusion condition (see Table 5A), when testing the reduction in BOLD signal for new compared to old (target and non target) items during the exclusion task under scopolamine, there were significant differences in the left superior (BA9) and middle (BA6) frontal gyri, the left perirhinal cortex and cingulate (see Table 6). No differences were found for old-new contrasts when considering individually the inclusion task under scopolamine and both tasks (inclusion and exclusion) under placebo.

We next examined both the placebo and scopolamine data, for correlations between individual subject behavioral performance, in terms of percentage accuracy for target and new items (inclusion condition), and target, non-target and new items (exclusion condition), in regions modulated by scopolamine (reported in Tables 5 and 6). We found statistically significant correlations (inverse) between behavioral performance during new item presentation in the exclusion condition under scopolamine and the percentage of signal change in left superior frontal gyrus (BA9) $[R=$ $-0.89, P<0.0001]$ and left perirhinal cortex (BA36) $[R=-0.82$, $P<0.0001]$. As these two areas both showed a significant correlation with behavioral performance for new items, we next conducted a further Spearman's test to determine whether the percentage signal change in BA 9 and 36 was correlated. We found a significant positive correlation $(R=0.68, P=0.004$; all significant correlations are shown in Fig. 3).

Table 5

Maxima of regions showing significant $(P<0.001$ uncorrected) BOLD signal changes that express an interaction between drug and exclusion task (A) and the contrast inclusion minus exclusion under scopolamine (B)

\begin{tabular}{|c|c|c|c|c|c|}
\hline \multirow[t]{2}{*}{ Brain region } & \multirow[t]{2}{*}{ Side } & \multirow[t]{2}{*}{ BA } & \multirow[t]{2}{*}{ Size } & Coordinates $(\mathrm{mm})$ & \multirow{2}{*}{$\begin{array}{l}\text { Peak } \\
Z \text { score }\end{array}$} \\
\hline & & & & $y$ & \\
\hline
\end{tabular}

(A) Interaction between drug and exclusion task

$\begin{array}{llllllll}\text { Sup frontal gyrus } & \text { L } & 9 & 93 & -12 & 52 & 34 & 4.25\end{array}$

$\begin{array}{llllllll}\text { Med frontal gyrus } & \mathrm{R} & 6,8 & 45 & 16 & 26 & 42 & 3.94\end{array}$

$\begin{array}{llllllll}\text { Med frontal gyrus } & \text { L } & 6 & 15 & -12 & 34 & 42 & 3.35\end{array}$

$\begin{array}{llllllll}\text { Mid frontal gyrus } & \text { L } & 6 & 27 & -18 & 2 & 64 & 3.53\end{array}$

$\begin{array}{llllllll}\text { Perirhinal cortex } & \text { L } & - & 18 & -18 & -24 & -24 & 3.89\end{array}$

$\begin{array}{llllllll}\text { Sup temporal gyrus } & \text { L } & 22 & 68 & -64 & -50 & 16 & 4.05\end{array}$

$\begin{array}{llllllll}\text { Inf parietal lobule } & \mathrm{R} & 5,7 & 57 & 38 & -38 & 34 & 3.83\end{array}$

$\begin{array}{llllllll}\text { Cingulate gyrus } & \text { L } & 24 & 22 & -10 & 12 & 36 & 3.75\end{array}$

$\begin{array}{llllllll}\text { Cingulate gyrus } & \mathrm{L} & 24 & 11 & -18 & 24 & 40 & 3.39\end{array}$

$\begin{array}{llllllll}\text { Cingulate gyrus } & \mathrm{R} & 31 & 16 & 14 & -56 & 24 & 3.67\end{array}$

$\begin{array}{llllllll}\text { Cingulate gyrus } & \mathrm{R} & 23 & 10 & 10 & -38 & 42 & 3.23\end{array}$

$\begin{array}{llllllll}\text { Cerebellum } & \mathrm{R} & - & 35 & 34 & -58 & -18 & 3.82\end{array}$

(B) Inclusion-scopolamine minus exclusion-scopolamine

$\begin{array}{llllrrrl}\text { Sup frontal gyrus } & \mathrm{L} & 9 & 74 & -12 & 52 & 34 & 4.06 \\ \text { Medial frontal gyrus } & \mathrm{L} & 10 & 16 & -8 & 56 & 0 & 3.36 \\ \text { Inferior frontal gyrus } & \mathrm{R} & 45,47 & 35 & 48 & 38 & 0 & 4.09 \\ \text { Perirhinal cortex } & \mathrm{L} & - & 20 & -18 & -24 & -24 & 4.05 \\ \text { Sup temporal gyrus } & \mathrm{L} & 22 & 83 & -64 & -56 & 14 & 4.05 \\ \text { Cingulate gyrus } & \mathrm{R} & 24,32 & 65 & 4 & 40 & 0 & 3.48 \\ \text { Cingulate gyrus } & \mathrm{L} & 32 & 16 & -18 & 24 & 38 & 3.39 \\ \text { Insula } & \mathrm{R} & - & 23 & 34 & -16 & 24 & 3.88 \\ \text { Fusiform gyrus } & \mathrm{R} & 37 & 26 & 34 & -60 & 18 & 3.82\end{array}$

Abbreviations: $\sup =$ superior; inf $=$ inferior; ant $=$ anterior; post $=$ posterior; mid =middle; med =medial; $\mathrm{R}=$ right $\mathrm{L}=$ left $\mathrm{B}=$ bilateral; $\mathrm{BA}=$ Brodmann area.

The extension of each area (size) is expressed in number of voxels. Only regions that were statistically significant at uncorrected level ( $\left.P_{\text {uncorrected }}<0.001\right)$ with a size of 10 or more voxels have been considered.

See text for further details. 

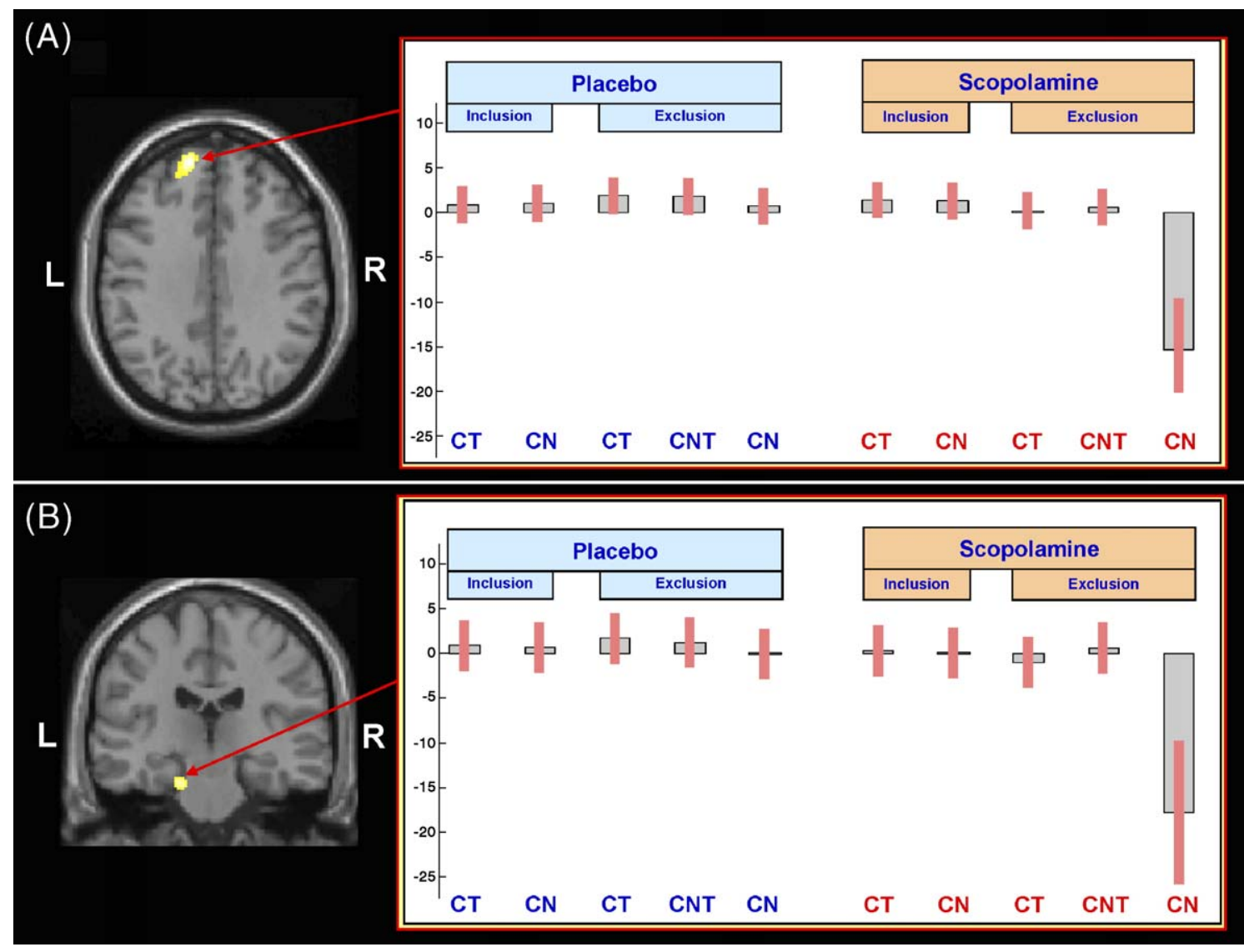

Fig. 2. Percentage BOLD signal change in each inclusion (target and new items) and exclusion subcondition (target, non-target and new items) under placebo and scopolamine for the most significant maxima identified in the left superior frontal gyrus (A) and perirhinal cortex (B). CT= correct responses for target items; $\mathrm{CNT}=$ correct responses for non-target items; $\mathrm{CN}=$ correct responses for new items. See the Results section for further details.

The imaging data used to produce the results presented above were globally scaled. The use of global scaling was motivated by the possibility that drug-induced changes in global activity might affect the comparison with normal controls. Nevertheless, global scaling may cause artifactual deactivations when there are extended regions responding to a particular condition. To exclude such a potential confound, correlations between the global signal

Table 6

Maxima of regions showing significant $(P<0.001$ uncorrected) BOLD signal changes that express activation reduction for new compared to old (target and non target) items in the exclusion task under scopolamine

\begin{tabular}{|c|c|c|c|c|c|c|c|}
\hline \multirow[t]{2}{*}{ Brain region } & \multirow[t]{2}{*}{ Side } & \multirow[t]{2}{*}{ BA } & \multirow[t]{2}{*}{ Size } & \multicolumn{3}{|c|}{ Coordinates (mm) } & \multirow{2}{*}{$\begin{array}{l}\text { Peak } \\
Z \text { score }\end{array}$} \\
\hline & & & & $x$ & $y$ & $z$ & \\
\hline Sup frontal gyrus & $\mathrm{L}$ & 9 & 46 & -14 & 50 & 34 & 3.73 \\
\hline Mid frontal gyrus & $\mathrm{L}$ & 6 & 23 & -18 & 0 & 64 & 3.60 \\
\hline Perirhinal cortex & $\mathrm{L}$ & - & 25 & -16 & -24 & -26 & 4.33 \\
\hline Cingulate gyrus & $\mathrm{L}$ & 24 & 36 & -8 & 12 & 36 & 4.09 \\
\hline
\end{tabular}

Abbreviations: $\sup =$ superior; inf $=$ inferior; ant $=$ anterior; post $=$ posterior; mid = middle; med =medial; $\mathrm{R}=$ right $\mathrm{L}=$ left; $\mathrm{B}=$ bilateral; $\mathrm{BA}=$ Brodmann area.

The extension of each area (size) is expressed in number of voxels. Only regions that were statistically significant at uncorrected level ( $\left.P_{\text {uncorrected }}<0.001\right)$ with a size of 10 or more voxels have been considered.

See text for further details. and the contrasts [inclusion minus exclusion], and [old minus new items] in the exclusion task, were tested separately for placebo and scopolamine. None of the four correlational values exceed \pm 0.1 (average across subjects), thus indicating that there was no systematic relationship between the global signal and the effects of interest.

\section{Discussion}

In the present study, we investigated the effects of scopolamine during complex source memory retrieval. The adapted paradigm was a modified version of the memory exclusion task of Jacoby (1996). We obtained a surprising set of findings for the effect of scopolamine during the exclusion condition, which were expressed when subjects responded to novel stimuli.

The study was set up to investigate the effect of scopolamine on prefrontal operations involved in source memory. The most surprising finding was a relative specificity in the modulation of a small set of brain regions through scopolamine and its highly specific relation to behavioral performance. Thus, scopolamine modulation effects were few as far as the interaction between drug effect and the inclusion condition was concerned. With the exception of the cerebellum, only two areas of the left inferior frontal gyrus were involved. Conversely, comparing the inclusion condition with baseline under placebo, the activation was mainly posterior (see Table 2). More critically, the signal change in these 
(A)

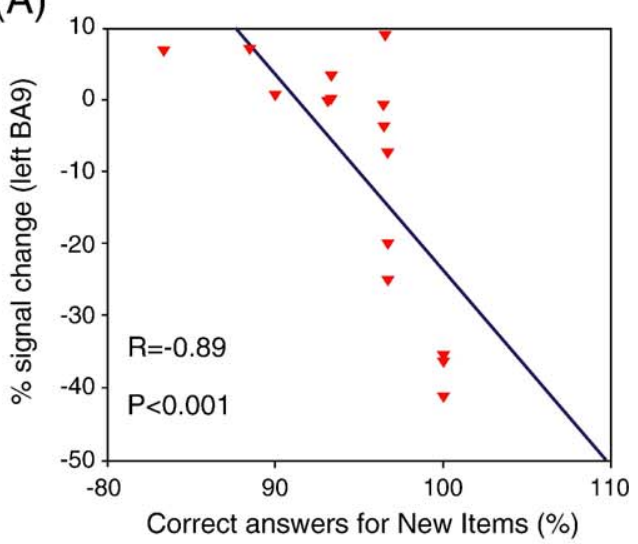

(B)

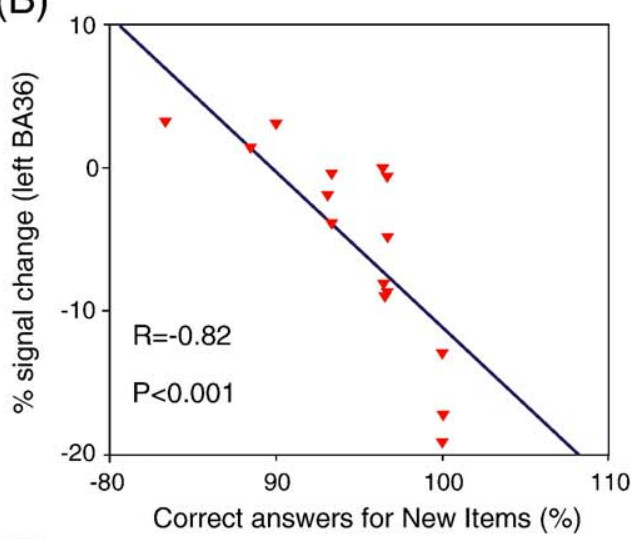

(C)

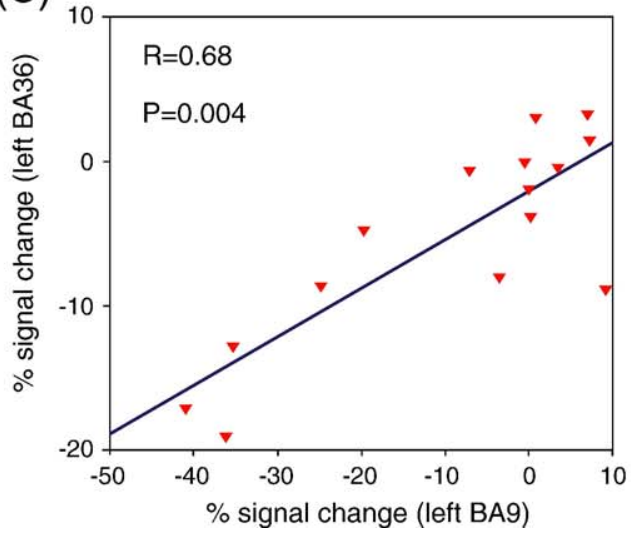

Fig. 3. Scatterplots of statistically significant correlations between individual subject behavioral performance on new items and the activation changes in left superior frontal gyrus (A) and perirhinal cortex (B) are shown. The positive correlation of percentage signal change between left superior frontal gyrus and perirhinal cortex is also shown (C). See the Results section for further details.

regions was not associated with any behavioral effect when performing the inclusion task.

A strikingly different pattern of activation and drug modulation was found when considering the exclusion task. Under placebo, there were widespread regions of activation in the exclusion condition compared to baseline (see Table 3). However, the interaction between drug and exclusion produced a more restricted set of regions, which showed greater difference in activation between exclusion and inclusion under scopolamine as compared to placebo (see Table 5); the critical regions being predominantly frontal and temporal. The region with the greatest $Z$ score - the left superior frontal gyrus (BA 9) - showed a distinctive activation pattern across the different response categories (target, non-target and new items), and this had a behavioral correlate. There was little difference in activation between scopolamine and placebo when participants correctly identified targets (old items in the same spatial position) or non-targets (old items in a different spatial position). Similarly, there was no difference between scopolamine and placebo in either the old or new conditions in the inclusion task. However, when participants responded to new items in the exclusion condition, there was a significant modulation with scopolamine compared to placebo (see Fig. 2A). Moreover, this modulation was inversely correlated with accuracy for new items across subjects (see Fig. 3A). This shows that effect is not a mere epiphenomenon created in the imaging analysis procedures. The second of these regions, left perirhinal cortex (BA 36), showed a similar effect both with respect to the pattern of drug modulation and the correlation with behavioral data (see Figs. 2B and 3B). Moreover, there was a direct correlation between the scopolaminerelated signal change in these two regions (see Fig. 3C).

How might this pattern of performance be explained? The deactivation occurs in the exclusion task only, not the inclusion task. This is the task where, for one group of stimuli, recollection and familiarity processes produce conflicting response tendencies. This suggests that the phenomenon may arise in the processes involved in the control of to what extent recollection and/or familiarity processes are operative. This explanation would require a second system or systems to control the relative strength of the familiarity and recollection signals, and so, the activity of perirhinal cortex. A candidate structure should have levels of activation that correlate with those of perirhinal cortex, as was the case for the left inferior frontal gyrus (BA9) in the current experiment (see Figs. 2 and 3). In fact, another study contrasting recollection and familiarity directly reported peak activation in a similar region only $1 \mathrm{~cm}$ distant. This study involved the Remember-Know paradigm, in which subjects must decide whether they can recollect having previously consciously experienced a stimulus or whether they simply find it familiar (Henson et al., 1999b). The region was deactivated during Know (familiarity) compared to Remember (recollection) responses.

What specifically could the control signal be doing? There seem to be two possibilities. One depends on the way the perirhinal cortex has been held by a number of authors to mediate a familiarity effect. Familiar stimuli lead to overall reduced neural activity in perirhinal cortex compared to novel stimuli (Aggleton and Brown, 2005). On this basis, new stimuli should evoke greater activation in perirhinal cortex than old stimuli do. If the effect of scopolamine is to produce a reduced activation in the left perirhinal cortex when new items are presented, the neural representation of new stimuli would masquerade as familiar stimuli. Therefore, we argue that, due to the effect of scopolamine on perirhinal cortex, it would be difficult under scopolamine to respond to an item as 'New' on the basis of a lack of a familiarity signature. Instead, the presence or absence of any recollection signal provides the sole basis for deciding whether a stimulus is in fact old or new. Thus, the system would need to base its response on recollection alone. By contrast, in the placebo condition, the judgment that an item is new can be based on familiarity as well as recollection. However, on this explanation, there is no simple account of why the effect occurs only in the exclusion and not also in the inclusion condition. 
A second possibility arises from the finding in the behavioral literature that it is impairments in acquisition rather than retrieval that are obtained under the administration of scopolamine (Ghoneim and Mewaldt, 1977; Petersen, 1977; Rosier et al., 1998). Acquisition effects operate on new stimuli or at least stimuli or stimulus combinations that are not yet well-learned. Thus, since the current phenomenon occurs in the processing of new stimuli, it might be seen as a side effect of acquisition processes. Moreover, it is known that, even when scopolamine affects encoding, it impairs subsequent free recall more than subsequent recognition (see Hasselmo and Wyble, 1997). This suggests - but does not demonstrate - that encoding for subsequent recollection-based judgments is more impaired than encoding for subsequent familiarity-based ones. The alternative possibility thus is the inverse of the first, namely that in the exclusion condition - which generally requires recollection, the New stimuli, specifically, are best processed under scopolamine using the familiarity signal rather than the recollection one and subjects doing so perform best. This is less of an issue for inclusion since there are no other stimuli for which the use of a recollection response is necessary. Moreover, in the Henson et al. (1999b) study, this region is deactivated for a 'Know', i.e. familiarity-based response. Thus, in this account, deactivation of perirhinal cortex is a sign of its being used!

The commonality between these two accounts is that they involve a control system which must oversee the selection of recollection and familiarity processes. Cholinergic-mediated involvement of the medial prefrontal cortex in this hypothetical memory control process may also relate to an analogous cholinergic role in associated structures - that is vigilance control in the rat (Dalley et al., 2004). Selecting between the two hypotheses would require further studies.

Another important issue in the study is whether the cholinergic input potentiates a monitoring process controlled by the dorsolateral prefrontal cortex. Our experimental paradigm involved a source memory - exclusion - condition that was argued by Henson et al. (1999a) to require this monitoring process. With certain exceptions, the overall pattern of results found by Henson et al. (1999a) was replicated. However, in the exclusion condition, the regions more activated compared to baseline were considerably more extensive than in the previous study, but included the main activated areas in the equivalent Henson et al. (1999a) contrast. One difference was that the anterior activations were more left lateralized in the current study, while they were more bilateral in the Henson et al. (1999a) study. This would fit with the findings of Dobbins et al. (2004) that monitoring based on spatial position, as in the current study, tends to be more left prefrontally based. In the Henson et al. (1999a) study, the source memory task also required knowledge of the list a word was presented in (temporal context), as well as its spatial position, but only the latter cue was relevant in the present study.

Turning to the effects of scopolamine, behaviorally it had little effect on accuracy across different conditions, with the exception discussed above (new item recognition in the exclusion condition). As far as the imaging results were concerned, the most important finding was that the set of regions involved in the interaction between drug and exclusion task did not overlap with the regions activated more in the exclusion task versus baseline contrast in the Henson et al. (1999a) study. Regions held to be involved in conscious monitoring or checking of the retrieval output, namely the mid-dorsolateral regions, particularly on the right, were not involved in the interaction between drug and exclusion task. There was therefore no support - either behaviorally or from imaging for the hypothesis that the basal forebrain cholinergic system might modulate the prefrontal structures responsible for monitoring the memory output. Therefore, this study provides no support for the hypothesis that impairments of this process due to lack of potentiation by the cholinergic system are involved in the causation of confabulations.

\section{Acknowledgment}

This work was supported by the Wellcome Trust.

\section{References}

Aggleton, J.P., Brown, M.W., 1999. Episodic memory, amnesia, and the hippocampal-anterior thalamic axis. Behav. Brain Sci. 22, 425-444 (discussion 444-489).

Aggleton, J.P., Brown, M.W., 2005. Contrasting hippocampal and perirhinal cortex function using immediate early gene imaging. Q. J. Exp. Psychol., B 58, 218-233.

Alexander, M.P., Freedman, M., 1984. Amnesia after anterior communicating artery aneurysm rupture. Neurology 34, 752-757.

Bond, A., Lader, M., 1974. The use of analogue scales in rating subjective feelings. Br. J. Med. Psychol. 47, 211-218.

Burgess, P.W., Shallice, T., 1996. Confabulation and the control of recollection. Memory 4, 359-411.

Cabeza, R., Locantore, J.K., Anderson, N.D., 2003. Lateralization of prefrontal activity during episodic memory retrieval: evidence for the production-monitoring hypothesis. Cogn. Neurosci. 15, 249-259.

Chudasama, Y., Dalley, J.W., Nathwani, F., Bouger, P., Robbins, T.W., 2004 Cholinergic modulation of visual attention and working memory; dissociable effects of basal forebrain 192-IgG-saporin lesions and intraprefrontal infusions of scopolamine. Learn. Mem. 11, 78-86.

Cipolotti, L., Bird, C., Good, T., Macmanus, D., Rudge, P., Shallice, T., 2006. Recollection and familiarity in dense hippocampal amnesia: a case study. Neuropsychologia 44, 489-506.

Cocosco, C.A., Kollokian, V., Kwan, R.K.S., Evans, A.C., 1997. BrainWeb: online interface to a 3D MRI simulated brain database. NeuroImage 5, S425.

Curran, T., DeBuse, C., Woroch, B., Hirshman, E., 2006. Combined pharmacological and electrophysiological dissociation of familiarity and recollection. J. Neurosci. 15, 1979-1985.

Dalley, J.W., Theobald, D.E., Bouger, P., Chudasama, Y., Cardinal, R.N., Robbins, T.W., 2004. Cortical cholinergic function and deficits in visual attentional performance in rats following 192 IgG-saporin-induced lesions of the medial prefrontal cortex. Cereb. Cortex 14, 922-932.

Damasio, A.R., Graff-Radford, N.R., Eslinger, P.J., Damasio, H., Kassell, N., 1985. Amnesia following basal forebrain lesions. Arch. Neurol. 42, 263-271.

DeLuca, J., 1993. Predicting neurobehavioral patterns following anterior communicating artery aneurysm. Cortex 29, 639-647.

DeLuca, J., Cicerone, K.D., 1991. Confabulation following aneurysm of the anterior communicating artery. Cortex 27, 417-423.

Dobbins, I.G., Simons, J.S., Schacter, D.L., 2004. FMRI evidence for separable and lateralized prefrontal memory monitoring processes. J. Cogn. Neurosci. 16, 908-920.

Fischer, R.S., Alexander, M.P., D’Esposito, M., Otto, R., 1995. Neuropsychological and neuroanatomical correlates of confabulation. J. Clin. Exp. Neuropsychol. 17, 20-28.

Fletcher, P.C., Henson, R.N., 2001. Frontal lobes and human memory: insights from functional neuroimaging. Brain 124, 849-881.

Ghoneim, M.M., Mewaldt, S.P., 1977. Studies on human memory: the interactions of diazepam, scopolamine, and physostigmine. Psychopharmacology 52, 1-6.

Gilboa, A., Moscovitch, M., 2002. The cognitive neuroscience of 
confabulation: a review and a model. In: Baddeley, A.D., Kopelman, M.D., Wilson, B.A. (Eds.), Handbook of Memory Disorders. John Wiley and Sons, London, pp. 315-342.

Hasselmo, M.E., Wyble, B.P., 1997. Free recall and recognition in a network model of the hippocampus: simulating effects of scopolamine on human memory function. Behav. Brain Res. 89, 1-34.

Hasselmo, M.E., Wyble, B.P., Wallenstein, G.V., 1996. Encoding and retrieval of episodic memories: role of cholinergic and GABAergic modulation in the hippocampus. Hippocampus 6, 693-708.

Henson, R.N.A., 2005. A mini-review of fMRI studies of human medial temporal lobe activity associated with recognition memory. Q. J. Exp. Psychol., B 58, 340-360.

Henson, R.N., Shallice, T., Dolan, R.J., 1999a. Right prefrontal cortex and episodic memory retrieval: a functional MRI test of the monitoring hypothesis. Brain 122, 1367-1381.

Henson, R.N., Rugg, M.D., Shallice, T., Josephs, O., Dolan, R.J., 1999b. Recollection and familiarity in recognition memory: an eventrelated functional magnetic resonance imaging study. J. Neurosci. 19, 3962-3972.

Hintzman, D.L., Caulton, D.A., Levitin, D.J., 1998. Retrieval dynamics in recognition and list discrimination: further evidence of separate processes of familiarity and recall. Mem. Cogn. 26, 449-462.

Hutton, C., Bork, A., Josephs, O., Deichmann, R., Ashburner, J., Turner, R., 2002. Image distortion correction in fMRI: a quantitative evaluation. NeuroImage 16, 217-240.

Hutton, C., Deichmann, R., Turner, R., Anderrson, J., 2004. Combined correction for geometric distortions and its interaction with head movement in fMRI. Proc. of the 12th Scientific Meeting and Exhibition of the ISMRM, p. 1084

Jacoby, L.L., 1996. Dissociating automatic and consciously controlled effect of study/test compatibility. J. Mem. Lang. 35, 32-52.

Lepage, M., Ghaffar, O., Nyberg, L., Tulving, E., 2000. Prefrontal cortex and episodic memory retrieval mode. Proc. Natl. Acad. Sci. U. S. A. 97, 506-511.

Mandler, G., 1980. Recognizing: the judgment of previous occurrence. Psychol. Rev. 87, 511-519.

Mesulam, M.M., 2000. Behavioural neuroanatomy: large scale networks; association cortex; frontal syndromes; the limbic system, and hemispheric specializations. In: Mesulam, M.M. (Ed.), Principles of
Behavioral and Cognitive Neurology. Oxford Univ. Press, New York, pp. 1-120.

Mintzer, M, Griffiths, RR, 2001. Acute dose-effects of scopolamine on false recognition. Psychopharmacology 153, 425-433.

Moscovitch, M., 1989. Confabulation and the frontal systems: strategic versus associated retrieval in neuropsychological theories of memory. In: RoedigerI.I.I. I.I.I., H.L., Craik, F.I.M. (Eds.), Varieties of Memory and Consciousness: Essays in Honour of Endel Tulving. Erlbaum, Hillsdale, NJ, pp. 133-160.

Petersen, R.C., 1977. Scopolamine induced learning failures in man. Psychopharmacology 52, 283-289.

Phillips, S., Sangalang, V., Sterns, G., 1987. Basal forebrain infarction. A clinicopathologic correlation. Arch. Neurol. 44, 1134-1138.

Roediger, H.L., McDermott, K.B., 1995. Creating false memoriesRemembering words not presented in lists. J. Exp. Psychol. Learn. 21, $803-814$

Rosier, A., Cornette, L., Orban, G.A., 1998. Scopolamine-induced impairment of delayed recognition of abstract visual shapes. Neuropsychobiology 37, 98-103.

Shallice, T., 2002. Fractionation of the supervisory system. In: Stuss, D.T., Knight, R.T. (Eds.), Principles of Frontal Lobe Function. Oxford Univ. Press, New York, pp. 261-277.

Shallice, T., Fletcher, P., Frith, C.D., Grasby, P., Frackowiak, R.S., Dolan, R.J., 1994. Brain regions associated with acquisition and retrieval of verbal episodic memory. Nature 368, 633-635.

Sperling, R., Greve, D., Dale, A., Killiany, R., Holmes, J., Rosas, H.D., Cocchiarella, A., Firth, P., Rosen, B., Lake, S., Lange, N., Routledge, C., Albert, M., 2002. Functional MRI detection of pharmacologically induced memory impairment. Proc. Natl. Acad. Sci. U. S. A. 99, 455-460.

Talairach, J., Tournoux, P., 1998. Co-planar Stereotaxic Atlas of the Human Brain. Thieme Medical Publishers, New York.

Tulving, E., Kapur, S., Craik, F.I., Moscovitch, M., Houle, S., 1994. Hemispheric encoding/retrieval asymmetry in episodic memory: positron emission tomography findings. Proc. Natl. Acad. Sci. U. S. A. 91, 2016-2020.

Yonelinas, A.P., Levy, B.J., 2002. Dissociating familiarity from recollection in human recognition memory: different rates of forgetting over short retention intervals. Psychon. Bull. Rev. 9, 575-582. 